Revista de Psicología Vol. 30 (2), 2012 (ISSN 0254-9247)

\title{
Modelado matemático del efecto del anclaje sobre el sesgo de subconfianza ${ }^{1}$
}

\author{
Guillermo Macbeth $^{2}$ y Eugenia Razumiejczyk ${ }^{3}$ \\ Universidad Nacional de Entre Ríos y CONICET, Argentina
}

Se define el efecto de anclaje como la tendencia del pensamiento humano a la fijación en determinados fragmentos de información que afectan la toma de decisiones. El sesgo de subconfianza, por su parte, se entiende como la propensión de un agente a subestimar su desempeño objetivo. Si bien se conocen algunos aspectos de la interacción entre ambos efectos, no se dispone de modelos formales que describan tal relación específica. Este estudio presenta: i) un experimento que pone en evidencia el efecto que ejerce el anclaje tanto para reducir, como para amplificar la subconfianza en tareas de decisión geográfica y ii) los fundamentos de un modelo matemático novedoso que permite estudiar con mayor precisión la relación del anclaje con la subconfianza.

Palabras clave: calibración, subconfianza, anclaje, sesgos heurísticos, modelado matemático

\section{Mathematical modelling of the anchoring effect on the underconfidence bias}

The anchoring effect is defined as the tendency of human thought to the fixation on certain pieces of information that affect decision-making. In the same context, the underconfidence bias is understood as an agent's propensity to underestimate his or her own objective performance. While some aspects of these phenomena are well known, there are no formal models that specifically describe the relationship between both. This paper presents: i) an experiment that illustrates the anchoring effect on the underconfidence bias by both reducing and amplifying such bias in the domain of geographic decision tasks and ii) the foundations of a new mathematical model that promotes precision in the study of the relationship between anchoring and underconfidence.

Keywords: calibration, under confidence, anchoring, biases, heuristics, mathematical modelling

La investigación informada en el presente artículo recibió financiación del Consejo Nacional de Investigaciones Científicas y Técnicas (CONICET) de Argentina mediante el proyecto de investigación plurianual PIP IU No 1142008010602 concedido al primer autor

2 Doctor en Psicología por la Universidad del Salvador, Argentina y Miembro de la Carrera del Investigador Científico del CONICET. Dirección Postal: Universidad Nacional de Entre Ríos, Facultad de Ciencias de la Educación (UNER-FCE). Alameda de la Federación 106, CP3100, Paraná, Entre Ríos, Argentina. Correo electrónico: g.macbeth@conicet.gov.ar

3 Doctora en Psicología por la Universidad del Salvador, Argentina y Miembro de la Carrera del Investigador Científico del CONICET. Correo electrónico: eugeniaraz@fibertel.com.ar 

El programa de investigación propuesto en la década de 1970 por Daniel Kahneman y Amos Tversky (2000; Kahneman, Slovic \& Tversky, 1982; Tversky \& Kahmenan, 1974) para el estudio de los sesgos y heurísticos del pensamiento humano ha logrado identificar varios fenómenos psicológicos relacionados con la toma de decisiones en situación de incertidumbre. Uno de los sesgos investigados por este programa es el efecto o sesgo de subconfianza o infraconfianza, que se define como la subestimación del éxito subjetivo en comparación con el éxito objetivo que presenta una persona en la realización de una colección de tareas (Gambara \& León, 1993; Garriga Trillo, Villarino, González Labra \& Arnau, 1994; Oskamp, 1965). El éxito subjetivo es entendido como la creencia personal acerca del propio desempeño, mientras que el éxito objetivo se refiere al puntaje efectivamente logrado en las tareas experimentales. La subconfianza ocurre cuando una persona cree o estima que su desempeńo ha sido peor que el rendimiento realmente alcanzado.

El efecto opuesto, conocido como efecto o sesgo de sobreconfianza, ha sido entendido como un optimismo exagerado en diversos contextos como la psicofísica (Garriga Trillo et al., 1994; Lubin \& Garriga Trillo, 1997), la clínica psicológica (Gambara \& León, 1993, 1996; Macbeth \& Bogiaizian, 2007), el asesoramiento económico (Camerer \& Lovallo, 1999), la resolución de problemas matemáticos (Macbeth, 2009b), la aptitud verbal (Macbeth \& Cortada de Kohan, 2008), la conducción de vehículos (Svenson, 1981) y la toma de decisiones judiciales (Farińa, Arce \& Novo, 2002), entre otros. En general, los fenómenos que surgen de la relación entre el éxito subjetivo o estimado $E$ y el éxito objetivo o rendimiento real $O$ se definen como calibración $C$, es decir, $C=\sum_{i=1}^{n} E_{i}-O_{i}$ cuando la tarea se compone de $n$ cantidad de ítems (Brenner, Koehler, Liberman \& Tversky, 1996; Klayman, 
Soll, Gonzalez-Vallejo \& Barlas, 1999; Oskamp, 1965). El sesgo de subconfianza surge cuando el valor de $C$ resulta negativo porque $E<O$. Sobre la base de esta definición básica se han desarrollado varios modelos formales estocásticos y no estocásticos de la calibración (Brenner, 2000, 2003; Erev, Wallsten \& Budescu, 1994; Gambara, Ruiz \& León, 1991; Macbeth \& Fernández, 2008; Merkle \& Van Zandt, 2006; Wallsten, Erev \& Budescu, 2000).

Tversky \& Kahneman (1974) mostraron experimentalmente hace casi cuatro décadas que los sesgos de la calibración pueden combinarse con otro fenómeno psicológico conocido como efecto de anclaje que también ha sido identificado por el mismo programa de investigación. Este fenómeno cognitivo ocurre cuando el proceso de decisión se concentra en un único criterio de información que ha sido provisto por alguno de los agentes involucrados en el contexto de la decisión. La información que promueve esta fijación cognitiva puede ser verdadera o falsa, completa o incompleta y funciona como un ancla que configura las decisiones. En estudios recientes sobre aptitud verbal y razonamiento informal se ha encontrado que los sesgos de la calibración pueden tanto moderarse o reducirse (Macbeth \& Fernández, 2008), como amplificarse (Macbeth, 2009a) mediante el anclaje. Una aplicación destacable de estos descubrimientos se informa en el estudio de Fariña et al. (2002) sobre la preponderancia del efecto del anclaje en las decisiones judiciales. Estos autores mostraron que las decisiones de los jueces penales de la población española incluida en su estudio están guiadas en más de un $60 \%$ por el anclaje en la pena solicitada por el ministerio fiscal, sin considerar otros elementos de relevancia jurídica.

En relación con estos antecedentes, los objetivos del presente estudio son: $i$ ) aumentar y disminuir la magnitud del sesgo de subconfianza mediante la manipulación del anclaje en tareas de decisión geográfica y; ii) proponer una descripción matemática que permita modelar el efecto del anclaje sobre el sesgo de subconfianza. Ambos objetivos son novedosos y buscan extender los conocimientos cognitivos actualmente disponibles sobre calibración. El objetivo $i$ se justifica porque el estado actual de los conocimientos específicos sobre este tema incluye tareas 
verbales y de razonamiento informal (Macbeth, 2009a), pero no se han realizado aún experimentos de amplificación y reducción de la subconfianza mediante anclaje en tareas que operen con materiales que resulten naturales o conocidos por los sujetos experimentales. Se seleccionaron por ello tareas de decisión geográfica relacionadas con la estimación del tamaño de poblaciones conocidas por los participantes del experimento como aproximación al estudio de las decisiones cotidianas. Si bien las decisiones de la tarea experimental propuesta no son estrictamente cotidianas, el material con el que operan puede ser considerado como conocido, natural o ingresado previamente en el sistema cognitivo.

En tal sentido, una novedad del presente trabajo radica en la extensión del estudio del efecto del anclaje sobre la calibración al dominio de la información geográfica, como complemento de estudios previos relacionados con los dominios del lenguaje y el razonamiento. El objetivo ii se justifica porque el programa de investigación de Kahneman y Tversky se propone no solo describir sesgos y heurísticos del pensamiento humano, sino también modelarlos matemáticamente (Kahneman et al., 1982). La abstracción de las explicaciones y su presentación en términos funcionales tanto estocásticos, como no estocásticos, es considerada como una herramienta útil para la descripción y el pronóstico del comportamiento de las decisiones humanas (Kahneman \& Tversky, 2000). Esta propuesta de formalización coincide, asimismo, con las sugerencias epistemológicas recientes de Rodgers (2010) referidas a la importancia del modelado matemático para la construcción de teorías psicológicas.

\section{Método}

En continuidad con los objetivos propuestos se presenta, en primer lugar, un experimento de calibración y, en segundo lugar, los fundamentos de un modelo matemático que permite describir los fenómenos estudiados en el experimento realizado. Se sugiere que este modelo provee una base formal para futuros experimentos y simulaciones computacionales 
capaces de extender, revisar y modificar las teorías psicológicas actuales sobre las estimaciones subjetivas de éxito afectadas por el anclaje. Las tareas experimentales del presente estudio solicitan tomar decisiones de dos opciones ante preguntas sobre magnitudes poblacionales de afinidad geográfica para los participantes. El modelo matemático propuesto, por su parte, consiste en la deducción de una función no estocástica específica que permite describir el comportamiento del sesgo de subconfianza en relación con el anclaje. Este modelo se ajusta al experimento presentado con tareas de decisión geográfica pero, adicionalmente, sugiere un algoritmo general para modelar diferentes estrategias de modificación de los sesgos de la calibración en otros dominios.

\section{Participantes}

Participaron en este experimento 360 estudiantes de grado de la carrera de Psicología de la Universidad de Buenos Aires y de la Universidad del Salvador, ambas situadas en la Ciudad Autónoma de Buenos Aires, Argentina. La participación femenina fue del 54\% (194 mujeres) y la masculina del 46\% (166 varones). La media de edad resultó de 24.97 años $(D T=7.10)$. Se estableció como criterio de exclusión de la muestra la condición de extranjero residente o no residente en Argentina por no cumplirse en este caso el ajuste entre las tareas propuestas y los conocimientos previos sobre la geografía local. Se excluyeron diez participantes de la muestra inicial por esta restricción. La asignación de los participantes a diferentes condiciones experimentales se realizó de manera aleatoria. Se mantuvieron proporciones similares de edad y sexo en todos los grupos. En todos los casos la participación fue libre, voluntaria y con consentimiento escrito.

\section{Instrumento}

Se administró a todos los participantes una prueba de rendimiento que incluye preguntas acerca de la magnitud poblacional de diferentes provincias argentinas. Esta prueba ha sido validada en estudios previos 
y posee adecuadas propiedades psicométricas (Macbeth \& Fernández, 2008). La tarea que propone la prueba consiste en indicar cuál de dos provincias argentinas dadas posee mayor cantidad de habitantes. Se presentan 66 pares de comparaciones que incluyen un total de 12 provincias. Cuatro son del norte, cuatro del centro y las cuatro restantes del sur de Argentina. En todos los casos se cumple que las provincias del centro son más populosas que las del norte. Las provincias del norte, a la vez, son siempre más populosas que las del sur. Las estadísticas poblacionales empleadas para el diseño del instrumento fueron obtenidas de los registros oficiales del Instituto Nacional de Estadística y Censos de Argentina (INDEC). Mediante el rendimiento en esta tarea se midió el éxito objetivo $O$. Para medir el éxito subjetivo $E$ se agregó al instrumento una pregunta final de estimación personal del éxito global alcanzado, según la cual cada participante indicó cuántas de las 66 comparaciones creyó responder de manera acertada.

Esta tarea resulta coherente con estudios previos sobre calibración que también utilizaron comparaciones de tamaños poblacionales, pero sin implementar en tales casos manipulaciones del anclaje (Brenner, 2003; Gambara et al., 1991; Gigerenzer, Hoffrage \& Kleinbölting, 1991; Macbeth, Ledesma, Razumiejczyk, Cortada de Kohan \& López Alonso, 2008).

\section{Procedimiento}

Se empleó un diseño completamente aleatorizado para comparar los efectos de diferentes anclajes sobre la calibración. Los participantes se asignaron aleatoriamente a uno de cinco grupos que fueron preparados para recibir diferentes tratamientos en fase de estudio y las mismas tareas y mediciones en fase de prueba. El grupo control no recibió ninguna manipulación. Se definieron cuatro grupos experimentales que recibieron anclajes de diferente dirección y magnitud. La dirección del anclaje se refiere a la variedad de sesgo de la calibración buscada experimentalmente, es decir, sobreconfianza $(\mathrm{E}>\mathrm{O})$ o subconfianza $(\mathrm{E}<\mathrm{O})$. La magnitud se refiere a la cantidad de desplazamiento fijada 
experimentalmente en el anclaje con el objetivo de obtener sesgos de la calibración de diferente tamańo del efecto. Debido a que la ausencia de anclaje en esta prueba generó una subconfianza intensa en estudios previos (Macbeth \& Fernández, 2008), con tamańos del efecto muy grandes $(d$ Cohen $>1.20)$, se propone en el presente estudio reducir este sesgo mediante tres niveles de incremento del anclaje y ampliarlo mediante un solo nivel de decremento.

Se consideró que la media de aciertos en estudios previos (Macbeth \& Fernández, 2008) resultó del 75\%, con aproximadamente 51 respuestas correctas $(D T \approx 6)$ sobre el total de 66 comparaciones. Para operacionalizar la manipulación del anclaje se indicó en la consigna de la tarea experimental que otras personas habían obtenido una determinada cantidad de respuestas correctas y que esta información debía tomarse en consideración antes de emitir la estimación de éxito subjetivo solicitada hacia el final de la prueba. Se utilizó tal estrategia para medir las variables $E, O$ y $C$ que conforman la definición operacional de la calibración (Gigerenzer et al., 1991). En la Tabla 1 se resumen las manipulaciones experimentales realizadas.

\section{Tabla 1}

Manipulaciones del anclaje en una tarea de calibración

\begin{tabular}{lccccc} 
& \multicolumn{5}{c}{ Grupo } \\
\cline { 2 - 6 } & Control & 1 & 2 & 3 & 4 \\
\hline Manipulación & ninguna & $\begin{array}{c}\text { anclaje } \\
\text { fuerte }\end{array}$ & $\begin{array}{c}\text { anclaje } \\
\text { fuerte }\end{array}$ & $\begin{array}{c}\text { anclaje } \\
\text { fuerte }\end{array}$ & $\begin{array}{c}\text { anclaje } \\
\text { débil }\end{array}$ \\
$\begin{array}{l}\text { \% de aciertos } \\
\text { declarados }\end{array}$ & - & $75 \%$ & $90 \%$ & $40 \%$ & $75 \%$ \\
$\begin{array}{l}\text { Cantidad } \\
\text { de aciertos } \\
\text { declarados }\end{array}$ & - & 50 & 60 & 26 & 50 \\
$\begin{array}{l}\text { Efecto buscado } \\
\text { Efención }\end{array}$ & - & $\begin{array}{c}\text { deducción } \\
\text { de sub- } \\
\text { confianza }\end{array}$ & $\begin{array}{c}\text { reducción } \\
\text { confianza }\end{array}$ & $\begin{array}{c}\text { amplificación } \\
\text { de sub- } \\
\text { confianza }\end{array}$ & $\begin{array}{c}\text { reducción } \\
\text { de sub- } \\
\text { confianza }\end{array}$ \\
\hline
\end{tabular}


En el grupo experimental 1 se implementó una manipulación de anclaje fuerte que consistió en incluir en la consigna cierta información sobre el rendimiento objetivo hallado en estudios previos, tal como se sugiere en estudios metodológicos que tratan sobre la manipulación de sesgos (Kahmenan \& Tversky, 1982). Se indicó que otros participantes obtuvieron unos 50 aciertos en la tarea propuesta. Esta manipulación es fuerte porque señala el rendimiento que otras personas tuvieron. La manipulación implementada en el grupo experimental 4 es, en cambio, débil porque indicó la cantidad de aciertos que otras personas han creido obtener. En el grupo experimental 2 se consignó un ancla de 60 aciertos que se corresponde con un $90 \%$ fuerte. En el grupo experimental 3 se fijó un ancla fuerte en la dirección opuesta, es decir, en el mismo sentido que la subconfianza. Se consignó que otras personas lograron unos 26 aciertos en promedio, lo cual se corresponde con un $40 \%$ de éxito objetivo. En los grupos experimentales 1, 2 y 4 se buscó reducir la magnitud de la subconfianza y en el grupo 3 se propuso amplificar este sesgo en el dominio de las decisiones geográficas, en coherencia con estudios previos que operaron sobre los dominios de la aptitud verbal y el razonamiento (Macbeth \& Fernández, 2008; Macbeth, 2009a).

\section{Hipótesis}

La hipótesis general de trabajo sostiene que el sesgo de subconfianza puede ser modificado mediante el anclaje y que esta relación admite una descripción funcional tratable matemáticamente. De esta manera, las hipótesis experimentales pronostican relaciones específicas entre la calibración y diferentes condiciones de manipulación del anclaje.

La hipótesis experimental $\mathrm{H} 1$ sostiene que la calibración en ausencia de manipulación incurre en el sesgo de subconfianza. Se espera que en el grupo control la media de éxito subjetivo $\bar{E}$, resulte significativamente menor que la media de éxito objetivo $\bar{O}$. Se propone expresar formalmente esta hipótesis como $H 1: \bar{E}_{\text {control }}<\bar{O}_{\text {control }}$. Se pronostica 
la generación de subconfianza en coherencia con estudios previos que utilizaron tareas de otros dominios, pero de dificultad similar a la del presente experimento (Macbeth \& Fernández, 2008; Macbeth \& Razumiejczyk, 2008; Macbeth, López Alonso, Razumiejczyk, Fernández, Sosa \& Pereyra, 2009).

La hipótesis $\mathrm{H} 2$ pronostica el sesgo de subconfianza para todas las condiciones de manipulación del anclaje. Formalmente, $H 2: \bar{E}_{i}<\bar{O}_{i}, \forall_{i}, i=\{1,2,3,4\}$. Esta hipótesis se justifica por estudios previos en los que se ha encontrado que solo el entrenamiento metacognitivo de dominio específico permite disolver de manera eficiente y sistemática los sesgos de la calibración (Macbeth \& Morán, 2009) y el anclaje implementado en este experimento no cumple tal condición.

La hipótesis $\mathrm{H} 3$ afirma que el sesgo de subconfianza se reduce en mayor medida por efecto de un anclaje fuerte que por efecto de un anclaje débil. Se espera que la información acerca de lo que otros lograron provoque mayor corrección del éxito subjetivo que la información acerca de lo que otros creyeron lograr. Formalmente, $H 3: \bar{C}_{\text {grupo } 1}<\bar{C}_{\text {grupo } 4}$. Se incluyó esta hipótesis por su relevancia para la implementación de programas correctivos en contextos aplicados (Farińa et al., 2002).

La hipótesis $\mathrm{H} 4$ afirma que el efecto de subconfianza se reduce por incremento del anclaje. Se pronostica que la información acerca del rendimiento incremental de otras personas genera correcciones graduales en la estimación subjetiva de éxito y reduce la magnitud de la distorsión. Formalmente, H4: $\bar{C}_{2}>\bar{C}_{1}>\bar{C}_{\text {control. }}$ La dirección de las inecuaciones en esta prueba de hipótesis se justifica por la operacionalización propuesta para la calibración $C$ en el presente estudio. Ésta variable arroja resultados negativos cuando el fenómeno observado es el sesgo de subconfianza, mientras que la ausencia de sesgos se corresponde con una $C$ nula. Debido a que se espera encontrar este fenómeno en todas las condiciones experimentales, se propone aquí operacionalizar la reducción del sesgo como una aproximación de $C$ a cero. Una apreciación visual de este fenómeno se presenta más abajo en la Figura 1, cuyo significado se comenta en la sección de Resultados. 
La hipótesis H5 sostiene que el efecto de subconfianza se amplifica por decremento del anclaje. Se espera que una manipulación en dirección opuesta a la reducción del sesgo provoque un incremento del mismo. Este efecto ha sido logrado previamente con tareas de lógica informal (Macbeth, 2009a). Formalmente, H5: $\bar{C}_{3}<\bar{C}_{\text {control }}$.

\section{Resultados}

Para garantizar la validez del experimento se requiere que las manipulaciones del anclaje afecten solo al éxito subjetivo $E$ pero no provoquen variaciones significativas del éxito objetivo $O$. Si esta condición no se cumple, entonces las diferencias de calibración $C$ resultan atribuibles a variaciones aleatorias de $O$ entre los grupos experimentales. Las diferencias observadas en $O$ resultaron atribuibles al azar $\left(F=0.554 ; p=.696 ; \eta_{p}^{2}=.006\right)$. Las diferencias observadas en $E$, en cambio, resultaron estadísticamente significativas con un tamaño del efecto grande $\left(F=17.023 ; p<.001 ; \eta_{p}^{2}=.161\right)$, por lo cual son atribuibles a las manipulaciones experimentales realizadas.

Las comparaciones entre las variables incluidas en las pruebas de hipótesis H1, H2, H3, H4 y H5 se resumen en la Tabla 2. Las distribuciones de frecuencias de estas variables resultaron compatibles con los supuestos de normalidad y homogeneidad de varianzas por las pruebas de Shapiro-Wilk y Levene, respectivamente, con $p$-valores mayores que .20 en todos los casos.

La hipótesis $\mathrm{H} 1$ resultó coherente con la evidencia experimental. Los participantes del grupo control incurrieron en el sesgo de subconfianza, en coherencia con estudios previos que emplearon pruebas similares (Macbeth \& Fernández, 2008).

La hipótesis $\mathrm{H} 2$ también resultó compatible con la evidencia. El sesgo de subconfianza persistió en todas las condiciones de anclaje utilizadas. El tamaño del efecto seńalado mediante la $d$ de Cohen (1988) en la Tabla 2 indica que la magnitud del sesgo varía entre diferentes condiciones experimentales. 


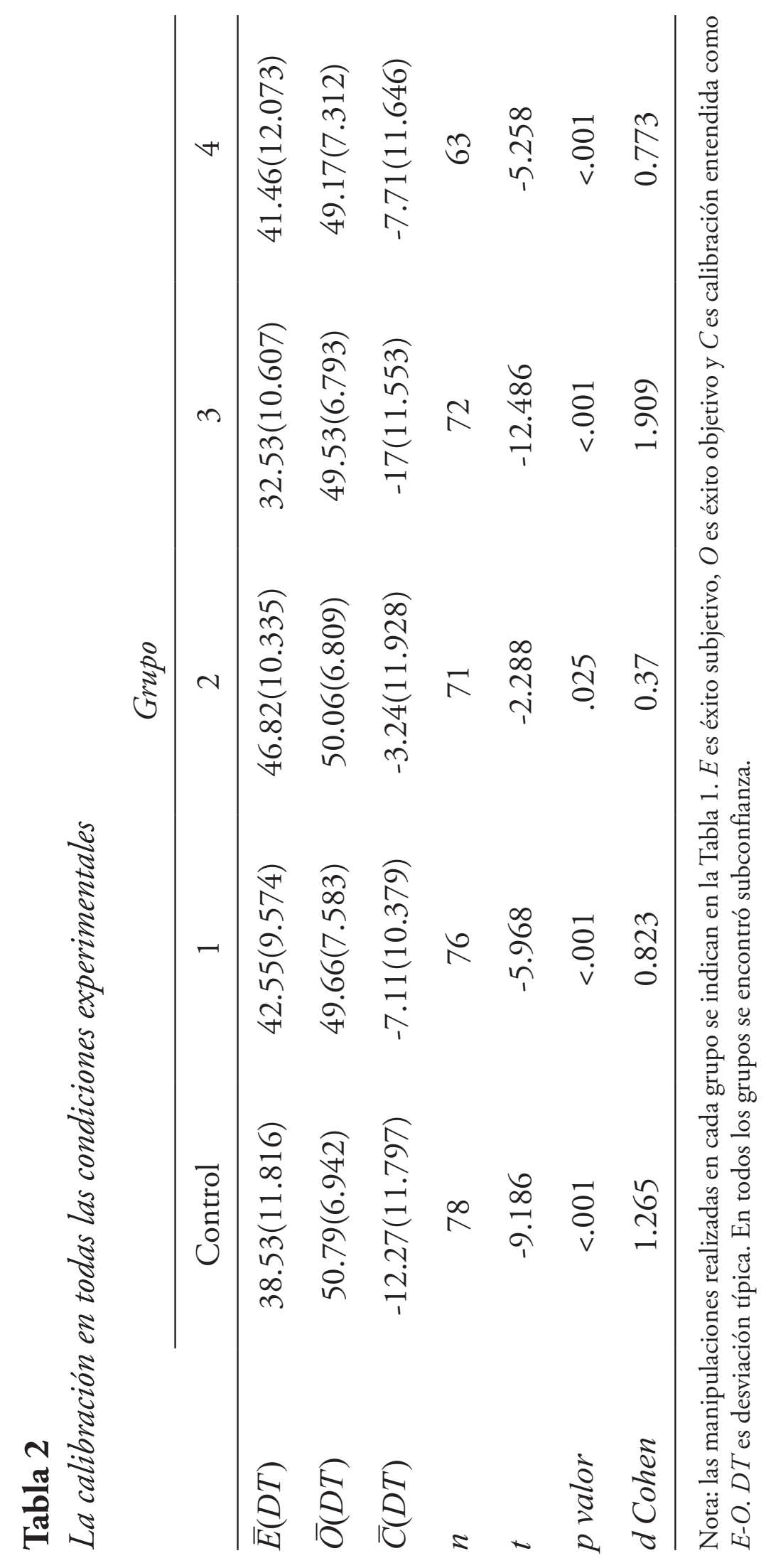


La hipótesis H3 resultó incompatible con la evidencia. No se encontró una diferencia significativa entre el anclaje fuerte y el anclaje débil fijado en un 75\% $(t=0.326 ; p=.745 ; d$ Cohen $=0.054)$. En ambos casos se logró la misma reducción parcial del sesgo. Esto sugiere que los participantes confiaron por igual en la información objetiva acerca del rendimiento de otras personas que en su testimonio subjetivo o personal. Este resultado posee implicancias sociales en tanto puede ser interpretado como una variedad de confianza en el juicio de otras personas, por la cual se asume que el testimonio ajeno se ajusta a la verdad sin estar expuesto a distorsiones de la calibración por subestimación o sobreestimación.

En coherencia con la hipótesis $\mathrm{H} 4$, el incremento del anclaje logró reducir la subconfianza $\left(F_{2,222}=11.843 ; p<.001 ; \eta_{p}^{2}=.096\right)$. Para el análisis post-hoc de las diferencias entre el grupo control y las dos condiciones experimentales se aplicó el método de Dunnett. Se encontraron diferencias significativas entre el control y el grupo $1(t=5.164 ; p<.05$; $d$ Cohen $=0.464)$ y entre el control y el grupo $2(t=9.03 ; p<.001$; $d$ Cohen $=0.761$ ), en coherencia con lo esperado. Este efecto reductor generado por el anclaje se aprecia en la Figura 1.

La hipótesis H5 resultó compatible con la evidencia. Se logró amplificar el sesgo por efecto de un anclaje orientado en la misma dirección que la subconfianza $(t=2.48 ; p=.014 ; d$ Cohen $=0.405)$. Este hallazgo extiende los conocimientos sobre la amplificación experimental de las distorsiones de la calibración al domino de tareas geográficas afines al ámbito de adaptación de los participantes. Tal resultado es coherente con las propuestas de estudios previos que han señalado la relevancia de la proximidad natural de los participantes a los materiales experimentales seleccionados (Dhami, Hertwig \& Hoffrage, 2004; Macbeth \& López Alonso, 2008). 


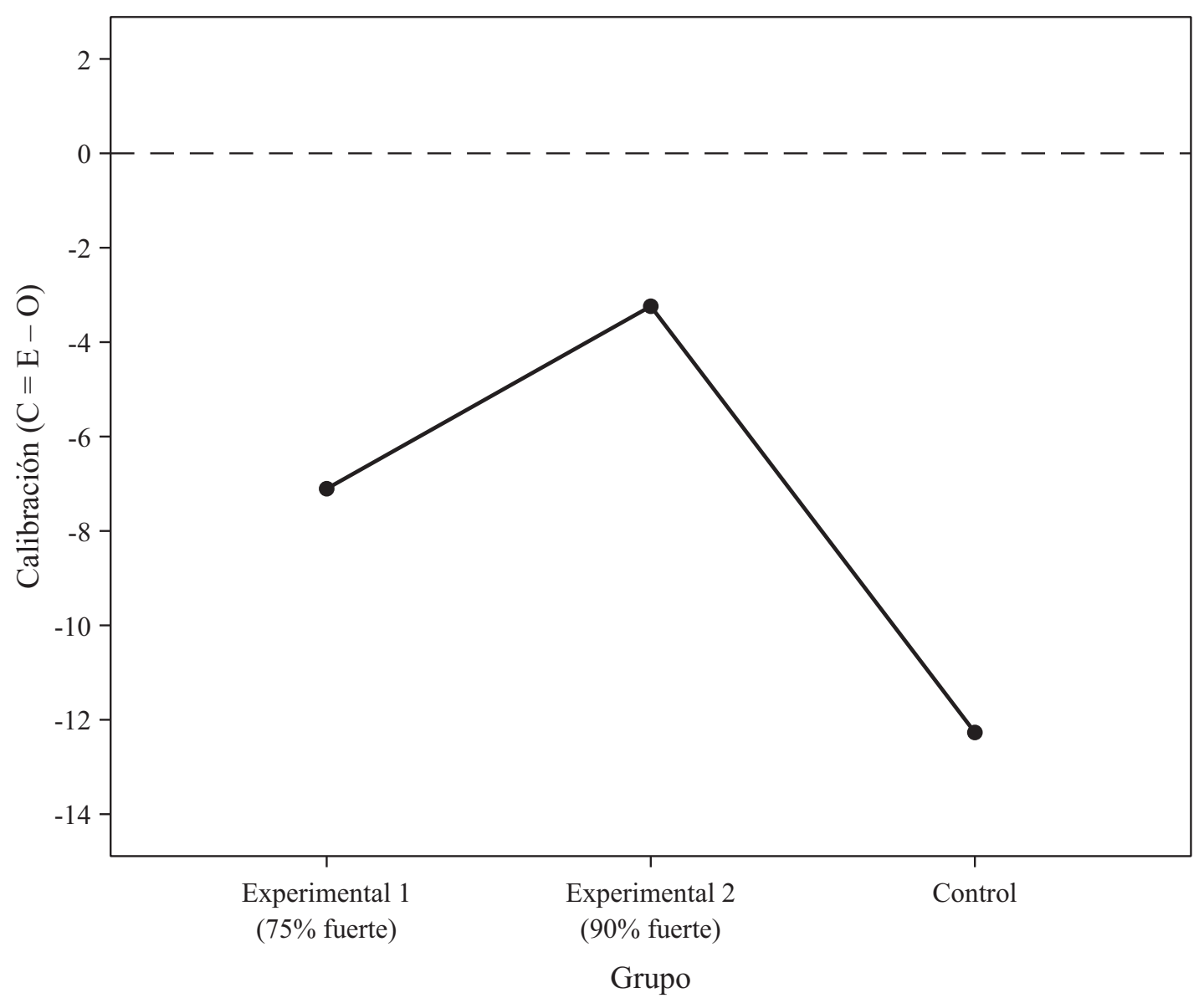

Figura 1. Efecto reductor del anclaje sobre el sesgo de subconfianza

La línea punteada en el cero de ordenadas indica la ausencia de sesgos. La aproximación a cero de los puntos correspondientes a los grupos experimentales es indicativa del efecto reductor del anclaje implementado.

\section{Discusión}

Los hallazgos de este experimento sugieren que el efecto de subconfianza puede ser modificado mediante la implementación de anclas que proveen información acerca de otros agentes. El efecto de anclaje permite tanto reducir, como amplificar las distorsiones de la calibración. Las variaciones generadas en la calibración pueden ser estudiadas en relación con la dirección y la fuerza del anclaje. Este resultado es coherente con hallazgos previos correspondientes a otros dominios y con el enfoque teórico que define la calibración como un fenómeno 
metacognitivo de monitoreo y control (Flavell, 1979; Nelson, 1996) que involucra la participación crítica de variables ambientales (Gigerenzer et al., 1991; Macbeth \& Morán, 2009; Macbeth, 2009a). Este enfoque sostiene que los sesgos de la calibración puede tanto moderarse y disolverse, como generarse y amplificarse por la manipulación de los procesos de monitoreo de la estimación subjetiva de éxito. El modelo de calibración propuesto en estudios previos por Macbeth y otros (Macbeth \& Fernández, 2008; Macbeth et al., 2009) sostiene, en el mismo sentido, que el incremento en el procesamiento de variables ambientales promueve calibraciones libres de sesgo. Las estrategias de modificación empleadas en este estudio son asimismo compatibles con las advertencias de Gigerenzer et al. (1991; Dhami et al., 2004) acerca del ajuste ecológico que requieren los experimentos de calibración. La extensión de hallazgos previos aquí propuesta se corresponde con tales desarrollos (Gigerenzer, 2000).

Una limitación del presente experimento consiste en la implementación de medidas globales para la medición del éxito subjetivo (Gambara \& León, 1993; Liberman \& Tversky, 1993). Junto con la tarea de estimación global de aciertos, se recomienda incluir además estimaciones ítem por ítem (Bersabé Morán, Martínez Arias \& Tejeiro Salguero, 2003). Si bien esta modalidad de operacionalización del éxito subjetivo ha recibido importantes críticas (Erev et al., 1994; Gambara \& León, 1996; Gigerenzer et al., 1991), se espera incluirla en futuras investigaciones por su relevancia para el estado del arte (Koehler, Brenner \& Griffin, 2002; Lichtenstein, Fischhoff \& Phillips, 1982). Otra limitación consiste en que las tareas de decisión que toman como criterio la estimación geográfica de magnitudes poblacionales no son tareas cotidianas que aporten evidencia específica para la discusión de los aspectos ecológicos de la calibración y el anclaje. Sin embargo, este experimento puede ser considerado como una aproximación a tal discusión porque la información sobre la que los participantes experimentales deciden es parcialmente conocida en tanto todos han sido expuestos a datos sobre magnitudes poblacionales locales en la educación escolar obligatoria. 


\section{Modelo matemático}

El programa de investigación propuesto por Tversky y Kahneman (1974) promueve la formulación de modelos matemáticos para describir y explicar el comportamiento de los sesgos y heurísticos de las decisiones humanas. Tal propósito es compatible con el logro de una mayor precisión, abstracción y genralidad en el análisis de los fenómenos de interés. Para describir el efecto del anclaje sobre el sesgo de subconfianza se propone en el presente estudio hallar una función no estocástica con anclajes en el dominio, magnitudes de la distorsión en el codominio y una regla específica de correspondencia entre ambos. Sea sesgo(ancla) la función expresada en (1).

$$
\operatorname{sesgo}(\text { ancla }): x \in \mathfrak{R}, x \in[-100,+100] \rightarrow y \in \mathfrak{R}, y \in[0,+\infty) \text {. }
$$

Donde ancla = porcentaje declarado en manipulación - porcentaje de éxito objetivo en situación control, es decir, la diferencia entre la manipulación empleada para generar experimentalmente el anclaje y el rendimiento obtenido en ausencia de manipulación, ambas variables expresadas en porcentajes. El incremento del anclaje genera porcentajes positivos y promueve la reducción de la subconfianza. El decremento, en cambio, produce porcentajes negativos, recorre la dirección opuesta y promueve la amplificación del sesgo. En el experimento aquí presentado se encontró que el sesgo de subconfianza se reduce notablemente cuando el ancla se fija en 60 aciertos sobre un total de 66 ítems. En este caso el porcentaje declarado en manipulación es de $(60 * 100) / 66 \approx 90 \%$. En estudios previos la media de aciertos espontáneos resultó de $(50 * 100) / 66 \approx 75 \%$, lo cual representa el porcentaje de éxito objetivo en situación control. Al definir de esta manera el dominio de la función se obtienen los valores de la Tabla 3.

Se propone una aproximación por mínimos cuadrados para hallar la recta que mejor ajusta los pares ordenados $(x, y)$ de la Tabla 3. Se excluye la información referida al control por no tener anclaje y la referida a la manipulación débil del $75 \%$ porque no aporta ninguna novedad respecto de la manipulación fuerte, tal como resultó anteriormente de la prueba de hipótesis de $\mathrm{H} 3$. 


\section{Tabla 3}

Elementos de la función propuesta

\begin{tabular}{ccc}
\hline $\begin{array}{c}\text { Grupo experimental } \\
\text { (manipulación) }\end{array}$ & $\begin{array}{c}\text { Variablex } \\
\text { Anclaje }\end{array}$ & $\begin{array}{c}\text { Variable y } \\
\text { Magnitud sesgo }\end{array}$ \\
\hline $1(75 \%$ fuerte $)$ & $\left(50^{*} 100\right) / 66 \approx 75-75 \approx 0$ & 0.823 \\
$2(90 \%$ fuerte $)$ & $\left(60^{*} 100\right) / 66 \approx 90-75 \approx+25$ & 0.37 \\
$3(40 \%$ fuerte $)$ & $\left(26^{*} 100\right) / 66 \approx 40-75 \approx-35$ & 1.909 \\
\hline
\end{tabular}

Nota: los valores del anclaje son porcentajes. La magnitud del sesgo corresponde a la $d$ de Cohen indicada en Tabla 2.

Sea $y$ un vector columna de $m$ filas con la forma $\left(\begin{array}{c}y_{1} \\ \vdots \\ y_{m}\end{array}\right), A$ una matriz de $m$ filas y 2 columnas con la forma $\left(\begin{array}{cc}1 & x_{1} \\ \vdots & \vdots \\ 1 & x_{m}\end{array}\right)$ y $\left(\begin{array}{c}b \\ m\end{array}\right)$ un vector columna donde $b$ es la ordenada al origen y $m$ es la pendiente $\frac{\Delta y}{\Delta x}$ de la recta buscada. Se tiene que $\left(\begin{array}{c}b \\ m\end{array}\right)=\left(A^{t} A\right)^{-1} A^{t} y$, donde $A^{t}$ es la matriz traspuesta de $A$ y $A^{-1}$ es la matriz inversa de $A$. En el Anexo 1 se presenta el cálculo completo correspondiente a la aplicación del modelo propuesto con los datos del experimento realizado. Los valores hallados permiten obtener la recta oblicua $y=m x+b$ de la expresión (2) que mejor ajusta el efecto del anclaje sobre el sesgo de subconfianza.

$$
\operatorname{sesgo}(\text { ancla })=-2.6012 * \text { ancla }+0.9462
$$

La Figura 2 presenta la función ajustada en la expresión (2) que modela el efecto del anclaje sobre la calibración, tal como resultó en el experimento realizado. 


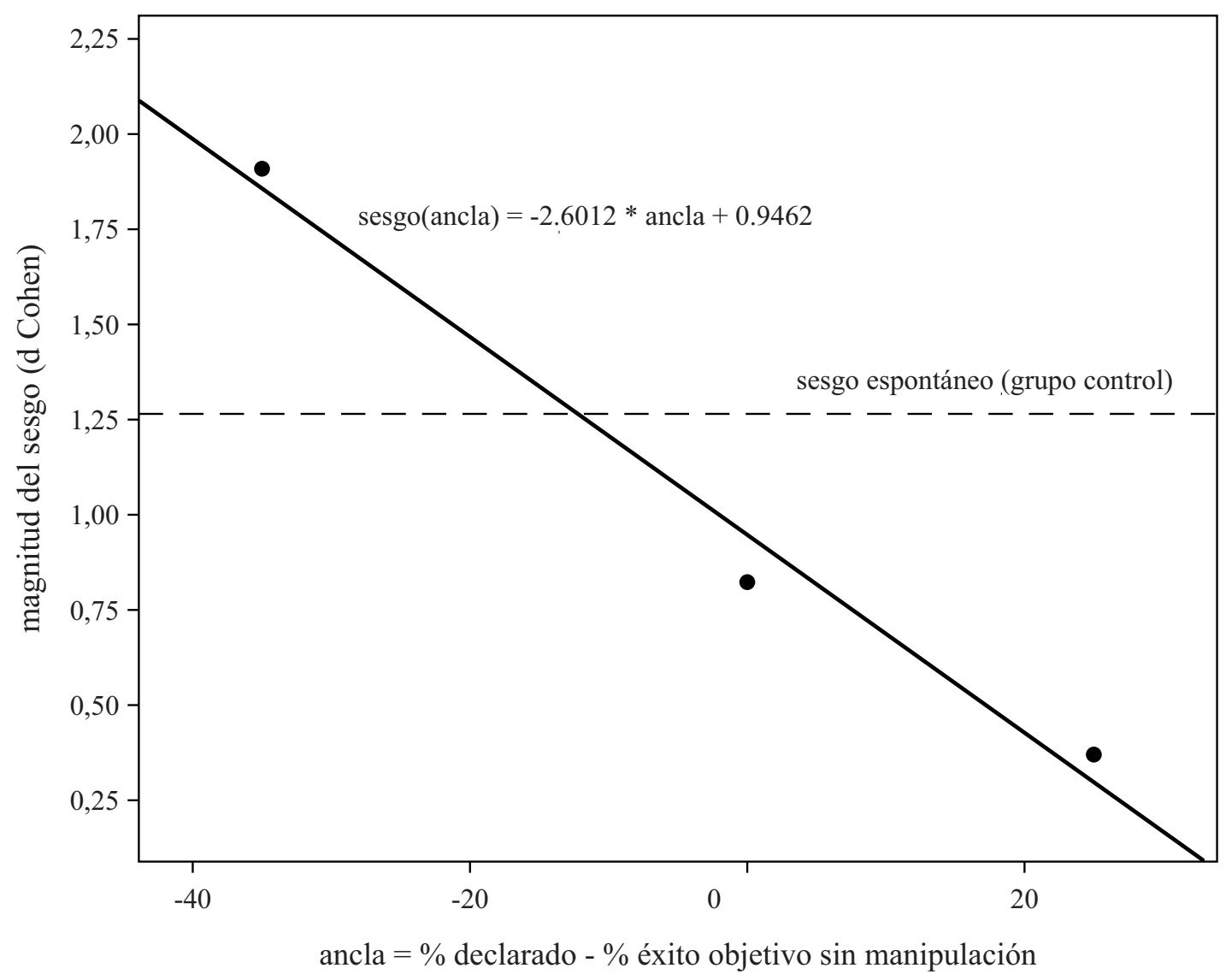

Figura 2. Efecto del anclaje sobre el sesgo de subconfianza

La línea horizontal discontinua indica la magnitud espontánea del sesgo de subconfianza y los puntos próximos a la recta oblicua representan los resultados experimentales del anclaje indicados en la Tabla 3. El punto que se encuentra por encima de la línea discontinua indica amplificación y los que están debajo indican reducción del sesgo de subconfianza. La magnitud correspondiente a una $d$ de Cohen $=1.265$ indica que el sesgo espontáneo de subconfianza es un efecto grande (Cohen, 1988). La pendiente pronunciada de la recta indica que la magnitud del sesgo presenta variaciones importantes en función del anclaje. La manipulación de anclas permite tanto aumentar, como disminuir la distorsión en tareas de decisión geográfica, en coherencia con estudios previos realizados en otros dominios (Macbeth, 2009a). 


\section{Discusión general}

Si bien se detectó tempranamente la relación entre los fenómenos de la calibración y el anclaje (Tversky \& Kahneman, 1974), solo a partir de los estudios de orientación ecológica (Gigerenzer et al., 1991) y de manipulación metacognitiva (Macbeth \& López Alonso, 2008) se comenzó a obtener evidencia sobre la posibilidad de moderación de los sesgos de sobreconfianza y subconfianza. En el primer ensayo histórico de revisión general sobre reducción de sesgos afirma Fischhoff (1982; Kahneman \& Tversky, 1982) que las distorsiones de la calibración son difíciles de remover. Una perspectiva similar se encuentra en revisiones más recientes (Kahneman \& Tversky, 2000; Wilson, Centerbar \& Brekke, 2002). La advertencia directa acerca de estos sesgos como parte de la consigna experimental, la inclusión de poblaciones expertas y la remuneración económica son estrategias de disolución que generalmente fracasaron. En cambio, la modificación del formato de la información para que su interpretación resulte más natural o espontánea para los participantes de los experimentos generó resultados favorables (Gigerenzer et al., 1991). La reformulación del concepto de calibración en términos ecológicos, es decir, mediante la inclusión de variables ambientales o de acceso natural para los sujetos experimentales, expandió la investigación de estos sesgos.

En estudios recientes se ha enfatizado, adicionalmente, la relevancia del procesamiento metacognitivo para la calibración (Macbeth \& Razumiejczyk, 2008). Se ha encontrado que solo las manipulaciones experimentales de nivel metacognitivo logran la disolución total de estas distorsiones (Macbeth \& Morán, 2009). Por metacognición se entiende el monitoreo y el control que una persona realiza sobre sus propios procesos cognitivos (Nelson, 1996). Los hallazgos del presente estudio resultan coherentes tanto con la perspectiva ecológica, como con el enfoque metacognitivo. En relación con el enfoque ecológico, se considera que el material experimental empleado presenta una relevancia ambiental parcial. Si bien las estimaciones de tamaños poblacionales no son decisiones cotidianas, la distribución geográfica 
local forma parte de la información escolar a la que todos los participantes experimentales han estado necesariamente expuestos. Esta condición posiblemente explica por qué se obtuvieron reducciones pero no disoluciones del sesgo de subconfianza. Se propone por ello buscar en futuros estudios la eliminación de sesgos de la calibración mediante el empleo de materiales estrictamente ecológicos, tal como lo sugiere la perspectiva metodológica iniciada por Egon Brunswik y Paul Meehl, entre otros, y promovida actualmente por Gerd Gigerenzer (Dhami et al., 2004).

Por otra parte, en relación con la metacognición, resulta relevante señalar que la manipulación del anclaje no es propiamente metacognitiva porque no procede por monitoreo y control, por lo cual no puede esperarse una disolución del sesgo. Sin embargo, la introducción experimental de información vinculada con el contexto de la decisión puede ser considerada como un sucedáneo del monitoreo en tanto consiste en la incorporación de nuevos datos al procesamiento cognitivo de la decisión. Tal condición es compatible con la reducción de sobreconfianza obtenida en el experimento y con la ausencia de disoluciones del sesgo. No se obtuvo la eliminación del sesgo porque no se implementó una manipulación metacognitiva plena, sino una variedad artificial de inclusión de información que comparte solo la novedad del dato con el monitoreo y el control metacognitivos propiamente dichos (Nelson, 1996). Se propone en este estudio que estas dos razones, la ecológica y la metacognitiva, permiten interpretar por qué el sesgo no se disolvió pero efectivamente se redujo, tal como se indicó en la Tabla 2.

El modelado matemático propuesto describe el comportamiento de la relación entre el anclaje y la magnitud del sesgo de subconfianza para el experimento realizado. La estrategia de asignación de dominio, codominio y regla de correspondencia que aquí se ha introducido proporciona adicionalmente una sugerencia general que resulta aplicable a diversos moderadores de la calibración. Este modelo es generalizable y susceptible de simulación mediante métodos tanto estocásticos, como no estocásticos. Una limitación del modelo consiste en la omisión 
de intervalos de confianza que permitan detectar sesgos espurios, tal como lo sugieren Gambara y León $(1993,1996)$ y Erev et al. (1994). Esta limitación se ha atendido parcialmente mediante la inclusión de medidas de tamaño del efecto que permiten controlar simultáneamente los errores de tipo I y II. Se requieren nuevas réplicas y estudios de simulación para evaluar si otras funciones polinómicas cuadráticas o cúbicas logran mejores ajustes que la función lineal propuesta. La acumulación de réplicas permitirá, asimismo, estabilizar estadísticamente los valores de los coeficientes hallados cuando se cumplen las condiciones del experimento presentado.

En el caso de restricción del procedimiento a dominios, tareas y poblaciones específicas, el modelo presenta interés aplicado para la reducción y posible eliminación de distorsiones de la calibración. La investigación de tal transferencia de aplicaciones ha sido sugerida para los campos profesionales de la Educación, la Economía, la Medicina y el Derecho, entre otros (Koehler et al., 2002). La introducción de modelos matemáticos de sesgos y heurísticos resulta asimismo coherente con las ventajas epistemológicas que ha señalado recientemente Rodgers (2010) relacionadas con la precisión, la generalidad y la aplicabilidad de las teorías psicológicas futuras.

\section{Referencias}

Bersabé Morán, R., Martínez Arias, R. \& Tejeiro Salguero, R. (2003). Risk-takers: Do they know how much of a risk they are taking? Psychology in Spain, 7(1), 3-9.

Brenner, L. (2000). Should observed overconfidence be dismissed as a statistical artifact? Critique of Erev, Wallsten, and Budescu (1994). Psychological Review, 107(4), 943-946.

Brenner, L. (2003). A random support model of the calibration of subjective probabilities. Organizational Behavior and Human Decision Processes, 90, 87-110. 
Brenner, L., Koehler, D. J., Liberman, V. \& Tversky, A. (1996). Overconfidence and probability and frequency judgments: A critical examination. Organizational Behavior and Human Decision Processes, 65(3), 212-219.

Camerer, C.F. \& Lovallo, D. (1999). Overconfidence and excess entry: An experimental approach. American Economic Review, 89(1), 306-318.

Cohen, J. (1988). Statistical power analysis for the behavioral sciences. Hillsdale, NJ: Lawrence Erlbaum \& Associates.

Dhami, M.K., Hertwig, R. \& Hoffrage, U. (2004). The role of representative design in an ecological approach to cognition. Psychological Bulletin, 130(6), 959-988.

Erev, I., Wallsten, T. S. \& Budescu, D. V. (1994). Simultaneous overand underconfidence: The role of error in judgment processes. Psychological Review, 101(3), 519-527.

Fariña, F., Arce, R. \& Novo, M. (2002). Heurístico de anclaje en las decisiones judiciales. Psicothema, 14(1), 39-46.

Fischhoff, B. (1982). Debiasing. En D. Kahneman, P. Slovic, \& A. Tversky (Eds.), Judgment Under Uncertainty: Heuristics and Biases (pp. 422-444). Cambridge: Cambridge University Press.

Flavell, J. H. (1979). Metacognition and cognitive monitoring. A new area of cognitive development inquiry. American Psychologist, 34(10), 906-911.

Gambara, H. \& León, O. G. (1993). Calibración en juicios clínicos. Revista de Psicología General y Aplicada, 46(1), 75-87.

Gambara, H. \& León, O. G. (1996). Subjective evidence of data and confidence in clinical judgements. European Journal of Psychological Assessment, 12, 193-201.

Gambara, H., Ruíz, M. A. \& León, O. G. (1991). Una simulación sobre la distribución muestral del índice de calibración. Psicológica, 13, 57-68.

Garriga Trillo, A. J., Villarino, A., González Labra, M. J. \& Arnau, M. A. (1994). La calibración de juicios psicofísicos: Estimación de magnitudes. Psicothema, 6(3), 525-532. 
Gigerenzer, G. (2000). Adaptive Thinking. Rationality in the Real World. Oxford: Oxford University Press.

Gigerenzer, G., Hoffrage, U. \& Kleinbölting, H. (1991). Probabilistic mental models: A brunswikian theory of confidence. Psychological Review, 98(4), 506-528.

Kahneman, D., Slovic, P. \& Tversky, A. (Eds.). (1982). Judgment under uncertainty: Heuristics and biases. Cambridge: Cambridge University Press.

Kahneman, D. \& Tversky, A. (1982). Intuitive prediction: Biases and corrective procedures. En D. Kahneman, P. Slovic, \& A. Tversky (Eds.), Judgment Under Uncertainty: Heuristics and Biases (pp. 414-421). Cambridge: Cambridge University Press.

Kahneman, D. \& Tversky, A. (Eds.). (2000). Choices, Values, and Frames. Nueva York: Cambridge University Press.

Klayman, J., Soll, J. B., Gonzalez-Vallejo, C. \& Barlas, S. (1999). Overconfidence: It depends on how, what and whom you ask. Organizational Behavior and Human Decision Process, 79, 216247.

Koehler, D.J., Brenner, L. \& Griffin, D. (2002). The calibration of expert judgment: Heuristics and biases beyond the laboratory. En T. Gilovich, D. Griffin \& D. Kahneman (Eds.), Heuristics and Biases. The Psychology of Intuitive Judgment (pp. 686-715). Cambridge: Cambridge University Press.

Liberman, V. \& Tversky, A. (1993). On the evaluation of probability judgments: Calibration, resolution and monotonicity. Psychological Bulletin, 114(1), 162-173.

Lichtenstein, S., Fischhoff, B. \& Phillips, L. D. (1982). Calibration of probabilities: The state of the art to 1980. En D. Kahneman, P. Slovic, \& A. Tversky (Eds.), Judgment Under Uncertainty: Heuristics and Biases (pp. 306-334). Cambridge: Cambridge University Press.

Lubin, P. \& Garriga Trillo, A. (1997). Efecto de la magnitud del estímulo sobre la confianza en los juicios psicofísicos. Psicothema, 9(2), 383-390. 
Macbeth, G. (2009a). Efecto amplificador del heurístico de anclaje sobre los sesgos de calibración. Acta Psiquiátrica y Psicológica de América Latina, 55(1), 3-12.

Macbeth, G. (2009b). Distorsiones del éxito subjetivo en la resolución de problemas matemáticos. Boletín de Psicología, 95(1), 59-72.

Macbeth, G. \& Bogiaizian, D. (2007). La estimación subjetiva de éxito en los trastornos de ansiedad. Revista Argentina de Clínica Psicológica, 16(2), 143-150.

Macbeth, G. \& Cortada de Kohan, N. (2008). Efecto del entrenamiento sobre la calibración subjetiva de éxito en tareas verbales. Revista Latinoamericana de Psicología, 40(1), 9-20.

Macbeth, G. \& Fernández, H. (2008). Moderadores metacognitivos del sesgo de subconfianza. Informes Psicológicos, 10(11), 189206.

Macbeth, G., Ledesma, R., Razumiejczyk, E., Cortada de Kohan, N. \& López Alonso, A.O. (2008). La medición de los sesgos de la calibración mediante modelos discretos y continuos. Investigaciones en Psicología, 13(1), 117-134.

Macbeth, G. \& López Alonso, A. O. (2008). Aportes de enfoque ecológico a los estudios sobre calibración. Acta Psiquiátrica y Psicológica de América Latina, 54(1), 55-61.

Macbeth, G., López Alonso, A. O., Razumiejczyk, E., Fernández, H., Sosa, R. A. \& Pereyra, C. I. (2009). Sesgos de la calibración en tareas de razonamiento lógico. SUMMA Psicológica UST, 6(2), 19-30.

Macbeth, G. \& Morán, V. (2009). El sesgo de subconfianza como fenómeno de dominio específico. Revista Latinoamericana de Psicología, 41(1), 47-57.

Macbeth, G. \& Razumiejczyk, E. (2008). Disolución del sesgo de subconfianza en tareas verbales. Anales de Psicología, 24(1), 143-149.

Merkle, E. \& Van Zandt, T. (2006). An application of the Poisson Race Model to confidence calibration. Journal of Experimental Psychology: General, 135(3), 391-408. 
Nelson, T. O. (1996). Consciousness and metacognition. American Psychologist, 51(2), 102-116.

Oskamp, S. (1965). Overconfidence in case-study judgments. The Journal of Consulting Psychology, 29, 261-265.

Rodgers, J. L. (2010). The epistemology of mathematical and statistical modeling: A quiet methodological revolution. American Psychologist, 65(1), 1-12.

Svenson, O. (1981). Are we all less risky and more skillful than our fellow drivers? Acta Psychologica, 47, 143-148.

Tversky, A. \& Kahneman, A. (1974). Judgment under uncertainty: heuristics and biases. Science, 185(4157), 1124-1131.

Wallsten, T. S., Erev, I. \& Budescu, D.V. (2000). The importance of theory: Response to Brenner (2000). Psychological Review, 107(4), 947-949.

Wilson, T. D., Centerbar, D. B. \& Brekke, N. (2002). Mental contamination and the debiasing problem. En T. Gilovich, D. Griffin \& D. Kahneman (Eds.), Heuristics and Biases. The Psychology of Intuitive Judgment (pp. 185-200). Cambridge: Cambridge University Press. 


\section{Anexo 1}

Ajuste de una función de anclaje y calibración para los resultados experimentalesa

$$
\begin{aligned}
& \text { Sea }\left(\begin{array}{l}
b \\
m
\end{array}\right)=\left(A^{t} A\right)^{-1} A^{t} y \\
& A=\left(\begin{array}{cc}
1 & x_{1} \\
\vdots & \vdots \\
1 & x_{m}
\end{array}\right)=\left(\begin{array}{cc}
1 & 0 \\
1 & +0.25 \\
1 & -0.35
\end{array}\right) \quad y=\left(\begin{array}{c}
y_{1} \\
\vdots \\
y_{m}
\end{array}\right)=\left(\begin{array}{c}
0.823 \\
0.37 \\
1.909
\end{array}\right) \\
& A^{t} A=\left(\begin{array}{ccc}
1 & 1 & 1 \\
0 & 0.25 & -0.35
\end{array}\right)\left(\begin{array}{cc}
1 & 0 \\
1 & 0.25 \\
1 & -0.35
\end{array}\right)=\left(\begin{array}{cc}
3 & -0.1 \\
-0.1 & 0.185
\end{array}\right) \\
& \left(A^{t} A\right)^{-1}=\left(\begin{array}{cc}
3 & -0.1 \\
-0.1 & 0.185
\end{array}\right)^{-1}=\left(\begin{array}{ll}
0.339 & 0.183 \\
0.183 & 5.505
\end{array}\right) \\
& A^{t} y=\left(\begin{array}{ccc}
1 & 1 & 1 \\
0 & 0.25 & -0.35
\end{array}\right)\left(\begin{array}{c}
0.823 \\
0.37 \\
1.909
\end{array}\right)=\left(\begin{array}{c}
3.102 \\
-0.57565
\end{array}\right) \\
& \left(\begin{array}{l}
b \\
m
\end{array}\right)=\left(A^{t} A\right)^{-1} A^{t} y=\left(\begin{array}{ll}
0.339 & 0.183 \\
0.183 & 5.505
\end{array}\right)\left(\begin{array}{c}
3.102 \\
-0.57565
\end{array}\right) \\
& \left(\begin{array}{l}
b \\
m
\end{array}\right)=\left(\begin{array}{l}
1.051578-0.10534595 \\
0.567666-3.16895325
\end{array}\right) \\
& \left(\begin{array}{l}
b \\
m
\end{array}\right)=\left(\begin{array}{c}
0.94623405 \\
-2.60128725
\end{array}\right) \\
& y=m x+b=-2.6012 x+0.9642 \\
& \Rightarrow \operatorname{sesgo}(\text { ancla })=-2.6012 * \text { ancla }+0.9642
\end{aligned}
$$

Enviado: 26 de febrero de 2012 Aceptado: 05 de mayo de 2012 